\title{
Numerical Entropy Production of Shallow Water Flows along Channels with Varying Width
}

\author{
Sudi Mungkasi \\ Department of Mathematics, Faculty of Science and Technology, Sanata Dharma University, \\ Mrican, Tromol Pos 29, Yogyakarta 55002, Indonesia, \\ Email:sudi@usd.ac.id
}

\begin{abstract}
We study the behavior of numerical entropy production of shallow water flows along channel by varying width. The topography is assumed to be horizontal, but the channel width can be in an arbitrary shape. The flows are governed by the system of shallow water equations. These equations are numerically solved using a finite volume method. The entropy of water flows are considered as the physical energy of the flows. The local truncation error of the entropy is defined to be the numerical entropy production, which has been successful as a smoothness indicator of solutions to the shallow water equations. In this paper, we present our study results on the relationship between numerical entropy production of the shallow water equations and the cell width of the finite volume method. We find that as the cell width is halved, the maximum value of the numerical entropy production is doubled.
\end{abstract}

Keywords: finite volume method, numerical entropy production, shallow water equation, varying width channel

\section{Introduction}

Shallow water flows occur in many situations, such as river, creek, flood inundation and even tsunami events. Therefore modeling and simulation of shallow water flows are useful. Some models of shallow water flows have been available, such as the Saint Venant system or also known as the system of shallow water equations ${ }^{1}$.

We consider the numerical entropy production of the shallow water equations, when they are solved using a conservative finite volume method. The numerical entropy production is the local truncation error of the entropy ${ }^{2}$. In this case it is defined for the shallow water equations. Initial investigation has been conducted by the author ${ }^{3}$. It was found that the numerical entropy production is an accurate smoothness indicator of the solution to the equations with varying width. In our previous work, we take the circular dam break problem as a test case.

In this paper, we extend our previous research [3] to get a more detailed behavior of the numerical entropy production. Moreover, we investigate the relationship between numerical entropy production of the shallow water equations and the cell width of the finite volume method. As a reference of the entropy definition, we follow the work of Balbas and Karni ${ }^{4}$.

The rest of the paper is structured as follows. In Section 2, we present the mathematical model for our problem. The method to solve the model is provided in Section 3. Research results are written in Section 4. Some concluding remarks are drawn in Section 5.

\section{Mathematical Model}

In this section we recall the mathematical model for shallow water flows that involve varying width in one dimension. The mathematical model is a system of two simultaneous partial differential equations representing the conservation of mass and the conservation of momentum. They are given by

$$
\begin{gathered}
(\sigma h)_{t}+(\sigma h u)_{x}=0 \\
(\sigma h u)_{t}+\left(\sigma h u^{2}+\frac{1}{2} g h^{2} \sigma\right)_{x}=g h^{2} \sigma_{x}
\end{gathered}
$$

where $x$ is the space variable and $t$ is time variable. Here $\sigma=\sigma(x)$ is the channel width, $h=h(x, t)$ is water depth, $u=u(x, t)$ is water velocity, and $g$ is the acceleration due to gravity. Note that the notation $b_{x}$ 
represents the derivative of $b$ with respect to $x$. In this paper we assume that the topography is horizontal, but the channel width can have arbitrary geometry.

\section{Numerical Method}

The mathematical model above can be solved using a finite volume method. We consider the model in the vector form

$$
\mathbf{q}_{t}+\mathbf{f}(\mathbf{q})_{x}=\mathbf{s}
$$

This equation (3) is a balance law. Here $\mathbf{q}$ is the vector of conserved quantities, $\mathbf{f}(\mathbf{q})$ is the flux vector and $\mathbf{s}$ is the source vector, which are given by

$$
\mathbf{q}=\left[\begin{array}{c}
\sigma h \\
\sigma h u
\end{array}\right], \quad \mathbf{f}(\mathbf{q})=\left[\begin{array}{c}
\sigma h u \\
\sigma h u^{2}+\frac{1}{2} g h^{2} \sigma
\end{array}\right], \quad \mathbf{s}=\left[\begin{array}{c}
0 \\
g h^{2} \sigma_{x}
\end{array}\right]
$$

respectively. With the vector form, the space domain is discretized in to a finite number of cells, where the $i$ th cell width is denoted by $\Delta x_{i}$. In the semi-discrete scheme, the finite volume method is written as

$$
\frac{d \mathbf{q}_{i}}{d t}+\frac{1}{\Delta x_{i}}\left(\mathbf{F}_{i+\frac{1}{2}}-\mathbf{F}_{i-\frac{1}{2}}\right)=\mathbf{s}_{i}
$$

Here $\mathbf{F}_{i+\frac{1}{2}}$ and $\mathbf{F}_{i-\frac{1}{2}}$ are the incoming and the outgoing fluxes relating to the $i$ th cell. The notation $\mathbf{s}_{i}$ symbolizes the discretization of the source at the $i$ th cell. More detailed explanation about finite volume methods can be found in the work of LeVeque [1].

Following Balbas and Karni [4], the shallow water equations (1) and (2) are endowed with an entropy function. The entropy function is given by

$$
\eta(x, t)=\sigma h\left(\frac{1}{2} u^{2}+\frac{1}{2} g h\right) \text {. }
$$

Furthermore, the shallow water equations (1) and (2) admit the entropy inequality

$$
\eta_{t}+\left[u\left(\eta+\frac{1}{2} g h^{2} \sigma\right)\right]_{x} \leq 0
$$

The inequality (6) is understood in the weak sense. The numerical entropy production is defined to be the local truncation error of the entropy. Puppo and Semplice [2] proved that for conservation laws, the numerical entropy production is large at rough regions and small at smooth regions. In fact this is also true for balance laws as we see in the next section.

\section{Numerical Results}

In this section, we provide numerical results for achieving our goal. We consider a circular (radial) dam break problem. It is worthwhile to note that the analytical solution to the dam break problem along a rectangular channel on a horizontal topography has been available in the literature. However, the analytical solution to the circular dam break problem has not been found by any researcher until this paper is written. Therefore, we solve the circular dam break problem numerically using the aforementioned finite volume method. The circular dam break problem is governed by the mathematical model for the shallow water flows with varying width.

The numerical settings are as follows. We use a second order accurate method, that is, we discretize the space domain with a second order method as well as the time domain with a second order time stepping. The van Leer limiter is applied to deal with unphysical oscillation. The van Leer limiter is defined by

$$
\sigma_{i}^{n}=\frac{|a| a+|b| b}{|a|+|b|}
$$

respectively, where $a$ and $b$ are given by

$$
a=\frac{Q_{i+1}^{n}-Q_{i}^{n}}{\Delta x} \text { and } b=\frac{Q_{i}^{n}-Q_{i-1}^{n}}{\Delta x} .
$$

Here the superscript $n$ represents the $n$th time step. The subscript $i-1, i, i+1$ denote the $i-1, i, i+1$ th cells. The variable $Q$ represents the quantity to be reconstructed. Unless otherwise stated, the number of cells for the computation is 1000 .

In this paper, we solve two scenarios of circular dam break problem. The first scenario is the circular dam break problem on wet domain. The second scenario is the circular dam break problem involving a dry area. In all simulation, we take the acceleration due to gravity to be $9.81 \mathrm{~m} / \mathrm{s}^{2}$.

The first scenario is as follows. We consider a circular reservoir with radius $50 \mathrm{~m}$. Water inside the dam is of the depth $10 \mathrm{~m}$, and outside the dam is of the depth $1 \mathrm{~m}$. Recall that this problem is governed by the mathematical model (1) and (2). This can be understood by considering the symmetry of the problem. The channel width is then given by the function $\sigma(x)=2 \pi x$. The domain is from the center of the circular dam, which is the Origin of the space to the space point 
$x=100 \mathrm{~m}$. Therefore, the water depth is $10 \mathrm{~m}$ for $x$ in the interval $[0,50]$ and 1 for $x$ in the interval [50,100] . The two dimensional initial condition for the water surface is illustrated in Fig. 1. This figure is generated using ANUGA software (http://anuga.anu.edu.au), a free and open source software developed by the Australian National University (ANU) and Geoscience Australia (GA).

Fig. 2 shows the typical simulation result of the circular dam break problem of the first scenario. In this figure, the stage, momentum and velocity are depicted for time equals to $0,0.5,1,1.5$, and 2 seconds after dam break event. Note that stage means the level of free water surface, momentum corresponds to the water discharge and velocity is obviously the speed of flow with a certain direction. Discontinuities appear in this simulation. Those discontinuities are shock waves.

The evolution of the water surface (stage) and its Numerical Entropy Production (NEP) are shown in from Fig. 3 to Fig. 6. Fig 3 illustrates the initial condition. The flows at time $0.5,1$, and 2 seconds after dam break are plotted in Fig. 4, Fig. 5 and Fig. 6 respectively. The numerical entropy production is able to detect the shock propagation accurately. Note that in these figures, we only plot half of the whole domain. Half of the domain is enough to show the accuracy of the numerical entropy production, as the other half is symmetrical.

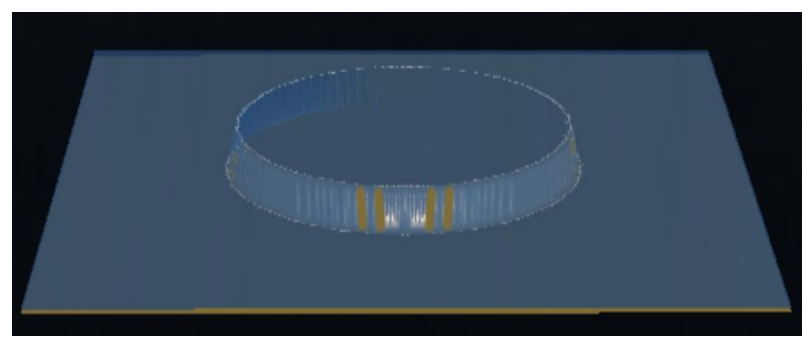

Fig. 1. The circular dam break problem at the initial condition. This figure is generated using ANUGA software (http://anuga.anu.edu.au).

We find that as the number of cells is doubled, the maximum value of the numerical entropy production is also doubled. This phenomena is true as the number of cells gets larger, as shown in Table 1. Consequently, we find the relationship between the numerical entropy production and the cell width.

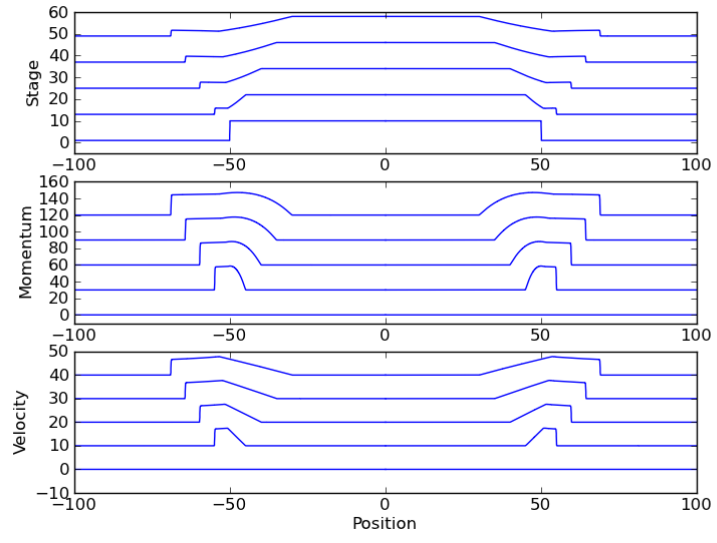

Fig. 2. Simulation results for time equals to $0,0.5,1,1.5$, and 2 seconds (five curves are plotted from below to above in each sub figure). Here the subfigures depict the stage, momentum and velocity.


Fig. 3. Initial condition for the water surface and its Numerical Entropy Production (NEP). 

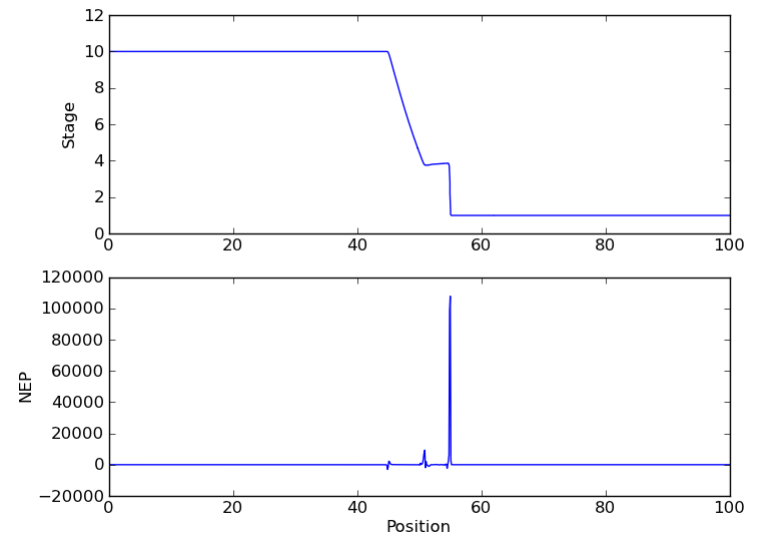

Fig. 4. The water surface (stage) and its Numerical Entropy Production (NEP) at time $t=0.5$.
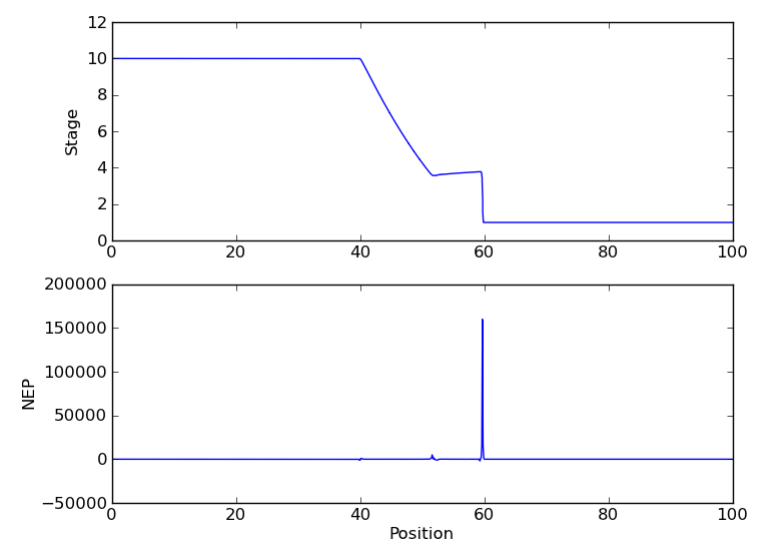

Fig. 5. The water surface (stage) and its Numerical Entropy Production (NEP) at time $t=1$.
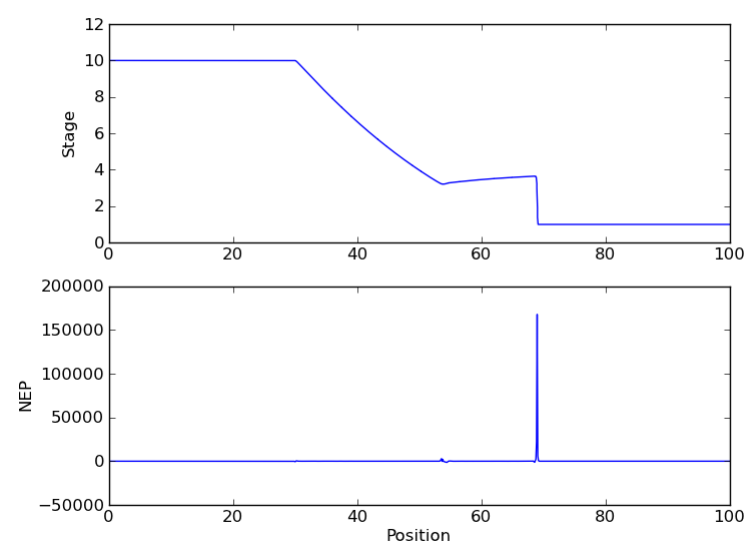

Fig. 6. The water surface (stage) and its Numerical Entropy Production (NEP) at time $t=2$.
Tabel 1. The relationship between the Numerical Entropy Production and the number of cells used in the computation. The results is at time $t=1$.

\begin{tabular}{cc}
\hline Number of cells & Maximum of NEP \\
\hline 100 & 13413 \\
200 & 35003 \\
400 & 49763 \\
800 & 144306 \\
1600 & 290497 \\
3200 & 576515 \\
6400 & 1133537 \\
\hline
\end{tabular}

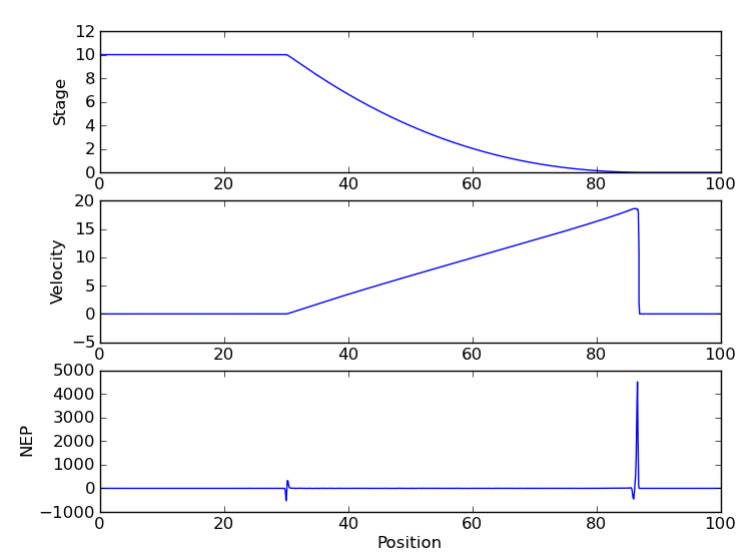

Fig. 7. The water surface (stage), velocity and its Numerical Entropy Production (NEP) at time $t=2$ for the second scenario.

Note that the number of cells is doubled means that the cell width is halved. Therefore, as the cell width is halved, the maximum value of the numerical entropy production is doubled.

Now, as the second scenario, we consider the following problem. We assume to have a circular reservoir with radius $50 \mathrm{~m}$, the same as before, with water inside the dam is of the depth $10 \mathrm{~m}$. However, water outside the dam is of the depth $10^{-6} \mathrm{~m}$. This very small number for the depth makes the area outside the reservoir to be considered "dry". Again, we use 1000 cells for our simulation. The results at time 2 seconds after dam break is viewed in Fig. 7. In this figure, the stage, momentum and its corresponding values of numerical entropy production are depicted. It is clear that the numerical entropy production is now successful in detecting the discontinuity of the velocity. 


\section{Conclusion}

We draw some remarks. Firstly, the numerical entropy production can detect discontinuities of the solution to the shallow water equations for flows along channels involving varying width. Secondly, the discontinuity is detected by a large value of the numerical entropy production. Thirdly, if the cell width is halved, the maximum value of the numerical entropy production is doubled. Finally, for future research we shall investigate the behavior of the numerical entropy production of the shallow water equations involving arbitrary topography and arbitrary channel width.

\section{References}

1. R. J. LeVeque, Finite-volume methods for hyperbolic problems, (Cambridge University Press, Cambridge, 2004).

2. G. Puppo and M. Semplice, Numerical entropy and adaptivity for finite volume schemes, Communications in Computational Physics 10(5) (2011) 1132-1160.

3. S. Mungkasi, An accurate smoothness indicator for shallow water flows along channels with varying width, submitted to Applied Mechanics and Materials.

4. J. Balbas and S. Karni, A central scheme for shallow water flows along channels with irregular geometry, ESAIM: M2AN 43(2) (2009) 333-351. 